\title{
THE HIGH ALTITUDE OBSERVATORY WHITE LIGHT CORONAGRAPH EXPERIMENT
}

R. M MacQUEEN, J. T. GOSLING, E. HILDNER, R. H. MUNRO,

\author{
A. I. POLAND, and C. L. ROSS
}

High Altitude Observatory, National Center for Atmospheric Research*, Boulder, Colo., U.S.A.

\begin{abstract}
The coronagraph has obtained observations, both by astronaut and ground command, spaced periodically throughout the mission on a time center of approximately six to eight hours. Programs of more frequent operation - to examine shortterm temporal variations and transient activity in the corona - have also been run at various times during the mission. The initial coronagraph observations of structures near limb passage experimentally verify that there are several time scales on which visual changes in these structures occur: (a) approximately one-half rotation, presumably accompanying major reorientations of coronal magnetic fields governing large scale coronal structures, (b) hours to days wherein changes to smaller coronal features are due either to structural changes of particular coronal features or to perspective effects and (c) less than hours - during coronal transients - which caused major reorientation of coronal structures by their passage through the coronal medium. Observations of the latter phenomena have provided some of the more spectacular results from the coronagraph (see Figure 1). The particular case illustrated was one stage of a complex series of events associated with activity in region 137, National Oceanic and Atmospheric Administration numbering, near east limb passage on 10 June 1973. The limb exhibited a major system of active prominences extended from the equator to north $25^{\circ}$ and produced surges and sprays through the early hours of the day. From approximately 0700 to 0900 UT several $\mathrm{H} \alpha$ and small $\mathrm{X}$-ray flares occurred and 0815 an eruptive prominence was observed by groundbased telescopes to ascend to $1.4 R_{\odot}$. This latter event is presumably the source of the transient observed by the coronagraph between 0929 and 1001 by ground command. During this period the material front moved with the apparent projected velocity of $450 \mathrm{~km} \mathrm{~s}^{-1}$ from 3.6 to $4.8 R_{\odot}$, while the arch-like structure expanded to a diameter greater than $2 R_{\odot}$. Figure 1 shows the event at $0943 \mathrm{GMT}$; it is one photograph of the 144 obtained. Within the structure are numerous bright areas and extended structures apparently mapping the distended magnetic field configuration. The general appearance of this transient is indicative of a large magnetic loop, or bottle, expanded outward from the Sun, its leading edge compressed by interaction with the ambient corona. Although there apparently was not a metric radio burst associated with this event, other transients observed later in the mission have associated metric wavelength activity.
\end{abstract}

* The National Center for Atmospheric Research is sponsored by the National Science Foundation.

Gordon Newkirk, Jr. (ed.) Coronal Disturbances, 505-506. All Rights Reserved.

Copyright C 1974 by the IAU. 


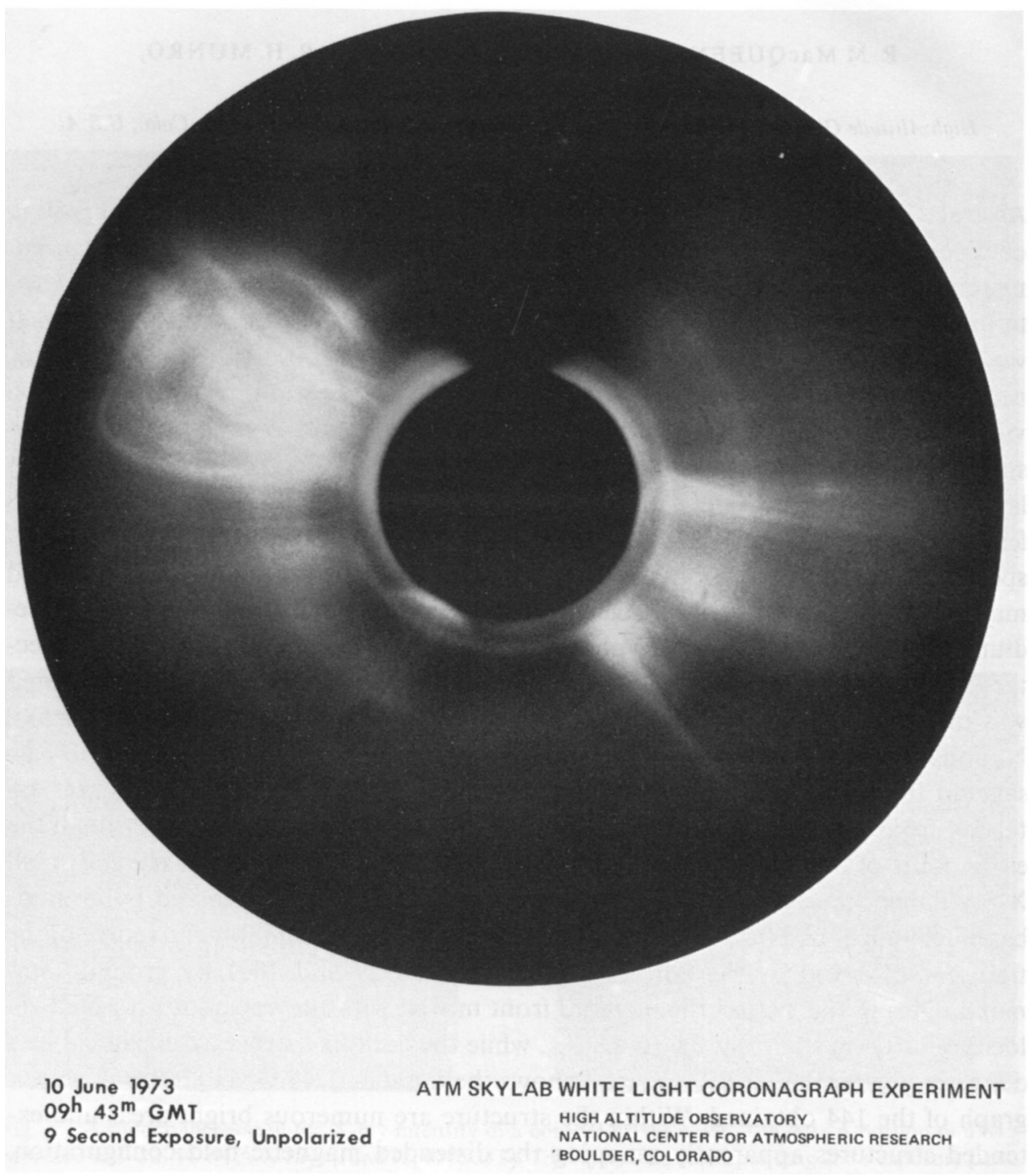

Fig. 1. The coronal transient of 10 June 1973. The photograph was made at 0943 GMT, more than one hour following ground observation of an eruptive prominence on the solar limb. Solar north is up, and east to the left. The coronagraph support pylon observes the northern solar corona, and present in the southeast is a cusp of scattered light due to contamination on the coronagraph external occulting disk. 